\title{
Microbial biotransformation: a process for chemical alterations
}

\begin{abstract}
Biotransformation is a process by which organic compounds are transformed from one form to another to reduce the persistence and toxicity of the chemical compounds. This process is aided by major range of microorganisms and their products such as bacteria, fungi and enzymes. Biotransformations can also be used to synthesize compounds or materials, if synthetic approaches are challenging. Natural transformation process is slow, nonspecific and less productive. Microbial biotransformations or microbial biotechnology are gaining importance and extensively utilized to generate metabolites in bulk amounts with more specificity. This review was conceived to assess the impact of microbial biotransformation of steroids, antibiotics, various pollutants and xenobiotic compounds.
\end{abstract}

Keywords: microbial biotransformation; bioconversion; metabolism; steroid; bacteria, fungi
Volume 4 Issue 2 - 2017

Smitha MS, Singh S, Singh R
Amity University Uttar Pradesh, India

Correspondence: Singh R, Additional Director, Amity Institute of Microbial Biotechnology, Amity University, Sector 125, Noida, UP, India, Tel +9 | I 204392900, Email rsingh3@amity.edu

Received: November 21, 2016 | Published: February 21, 2017

\section{Introduction}

Biotransformations are structural modifications in a chemical compound by organisms /enzyme systems that lead to the formation of molecules with relatively greater polarity. ${ }^{1,2}$ This mechanism has been developed by microbes to acclimatize to environmental changes and it is useful in a wide range of biotechnological processes. ${ }^{3}$ The most significant aspect of biotransformation is that it maintains the original carbon skeleton after obtaining the products. ${ }^{4}$

Biotransformation is of two types: Enzymatic and Non-enzymatic. Enzymatic are further divided into Microsomal and Non-microsomal. ${ }^{5}$ Enzymatic Elimination is the biotransformation occurring due to various enzymes present in the body. Microsomal biotransformation is caused by enzymes present within the lipophilic membranes of smooth endoplasmic reticulum. ${ }^{6}$ Non-Microsomal Biotransformation involves the enzymes which are present within the mitochondria. Examples include: Alcohol dehydrogenase responsible for metabolism of ethanol into acetaldehyde and Tyrosine hydrolases enzymes, Xanthine oxidase converting hypoxanthine into xanthine etc. Spontaneous, noncatalyzed and non-enzymatic types of biotransformation are for highly active, unstable compounds taking place at physiological $\mathrm{pH}$. Some of these include Chlorazepate converted into Desmethyl diazepam, Mustin $\mathrm{HCl}$ converted into Ethyleneimonium, Atracurium converted into Laudanosine and Quartenary acid, Hexamine converted into Formaldehyde.

Microbial biotransformation is widely used in the transformation of various pollutants or a large variety of compounds including hydrocarbons, pharmaceutical substances and metals. ${ }^{7}$ These transformations can be congregated under the categories: oxidation, reduction, hydrolysis, isomerisation, condensation, formation of new carbon bonds, and introduction of functional groups. ${ }^{8}$ For centuries microbial biotransformation has proved to be an imperative tool in alleviating the production of various chemicals used in food, pharmaceutical, agrochemical and other industries.

In the field of pharmaceutical research and development, biotransformation studies have been extensively applied to investigate the metabolism of compounds using animal models. ${ }^{9}$ The microbial biotransformation phenomenon is then commonly employed in comparing metabolic pathways of drugs and scaling up the metabolites of interest discovered in these animal models for further pharmacological and toxicological evaluation. ${ }^{10}$

The White biotechnology involves the use of microbial biotransformation for generating products of interest. ${ }^{11}$ Living cells such as bacteria, filamentous fungi, animals, plants, algae, yeast and actinomycetes are used. Microbial cells are ideal choice for biotransformation due to certain reasons like:

I. Surface-volume ratio: Microbial biotransformation has high surface-volume ratio.

II. Growth Rate: Higher growth rate of microbial cells reduces the time of biomass transformation.

III. Metabolism Rate: Higher rate of the metabolism in microbes leads to efficient transformation of substrate.

IV. Sterility: It is easier to maintain sterile conditions when microbes are used. ${ }^{12}$

\section{Procedure for biotransformation}

Vegetative cells, spores, resting cells, enzymes, and immobilized cells/enzymes are generally used for microbial transformation. ${ }^{13}$ In process with growing cultures, the strain used is cultivated in a suitable medium and a concentrated substrate solution is added after suitable growth of the culture $(6-24 \mathrm{~h})$. A variant of this procedure is to use a very large inoculum and to add the concentrated substrate immediately without allowing for a growth period. Emulsifier such as Tween or solvents (water-miscible and low toxic (ethanol, acetone, dimethyl formamide, dimethyl sulfoxide) may be used to help solubilise poorly soluble compounds. In some steroid transformations, the substrate is added and converted in fine crystalline form. These so-called pseudo-crystalline fermentations can be carried out with relatively high concentrations of substrate. ${ }^{14}$ 
For the biotransformation of lipophilic materials it is possible to employ a polyphase system. The aqueous phase containing the cell material or the enzyme is overlayed with the water immiscible fluid phase in which the substrate has been dissolved. The substrate passes slowly into the aqueous phase and as the transformation reaction proceeds, the product passes back into the solvent phase. In some cases, the actual transformation occurs at the interface of the aqueous and solvent phases.

Transformation reactions in large-scale equipment are carried out under sterile conditions in aerated and stirred fermenters, the conversion process being monitored chromatographically or spectroscopically. The process is terminated when a maximum titer is reached. Maintenance of sterile environment is necessary because contamination can lead to formation of faulty conversion products and can also engage in total breakdown.

Resting cells can be used in case if enzyme induction by the added substrate is not necessary. This has the considerable advantage that growth inhibition by the substrate is eliminated. High cell densities, which promote increased productivity, may be used; at the same time risk of contamination is reduced. Since the transformation reaction occurs predominantly in the buffer solution, the recovery of the product is relatively easy. A number of transformation processes employ immobilized cells, offering the advantage that the process can be carried out continuously and the cells can be used over and over again. ${ }^{15}$ Immobilized bacterial cells, which catalyse one-stage or multi-stage reactions, are presently used commercially in the production of aspartic acid, L-alanine and malic acid.

The end products of transformation reactions are found extracellularly and may occur in either dissolved or suspended form. The cell material is then washed repetitively with water or organic solvents in order to detach the reaction product which can be adsorbed to the cells. Depending on the solubility of the product, recovery is performed by adsorption to ion exchangers, by precipitation as the calcium salt, by extraction with appropriate solvents or for volatile substances, by direct distillation from the medium.

\section{Various applications of microbial biotransformation}

\section{Transformation of steroids and sterols}

Steroids constitute a natural product class of compounds that is widely distributed throughout nature present in bile salts, adrenalcortical and sex-hormones, insect molting hormones, sapogenins, alkaloids and some antibiotics. ${ }^{16}$ In 1937 the first microbial biotransformation of steroids was carried out. ${ }^{17}$ Testosterone was produced from dehydroepiandrosterone by using Corynebacterium $s p$. Subsequently cholesterol was produced from 4-dehydroeticholanic and 7-hydroxycholestrol using Nocardia spp.

All steroids have the same basic structure, a cyclopentanoperhydrophenanthrene which consists of four fused rings (Figure 1). ${ }^{18}$ Cortisone is very useful because of its anti-inflammatory action against rheumatold arthritis and skin diseases. By changing the structure, specifically by incorporating a 1,2 double bond in ring A of the cortisone molecule to produce prednisone which have the property of markedly increased anti-inflammatory effect.

The steroid molecule has several asymmetric centres and it makes the total synthesis of steroid very difficult. ${ }^{19}$ Preliminary research on the 11 alpha-hydroxylation of progesterone pointed to the possibility of the microbial introduction of oxygen into the steroid nucleus in a site specific and stereospecific manner without prior activation (Figure 2). These reactions worked well and cost-effective production of cortisone became possible. ${ }^{20}$ The above microbial step reactions are of great economic significance. Progesterone transformation of a C-19 steroid is used industrially in the production of testosterone and estrogen and the microbial dehydration of ring $\mathrm{A}$ is used in estrogen production.

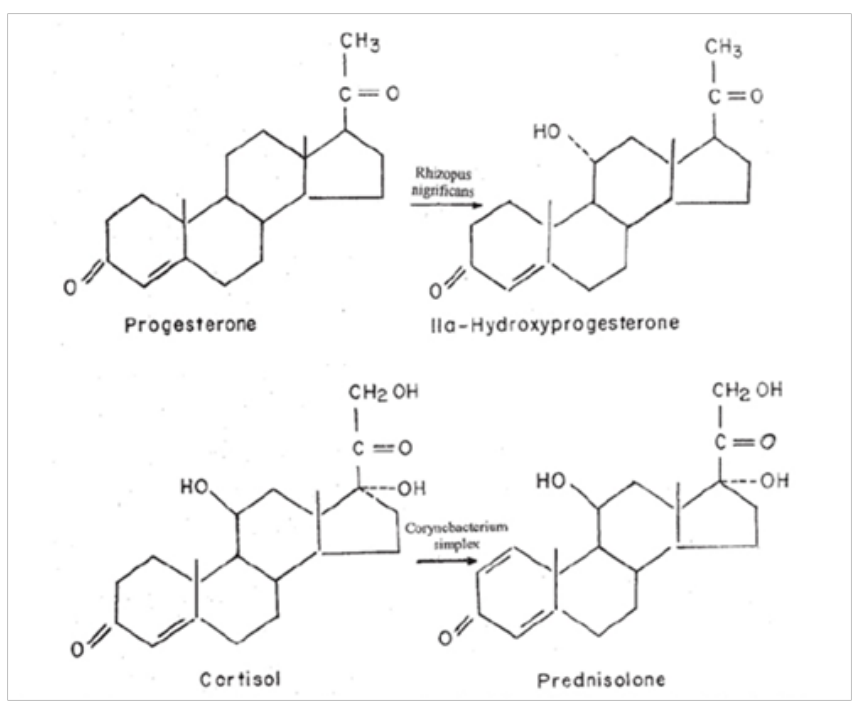

Figure I Biotransformation of commercially important steroids.

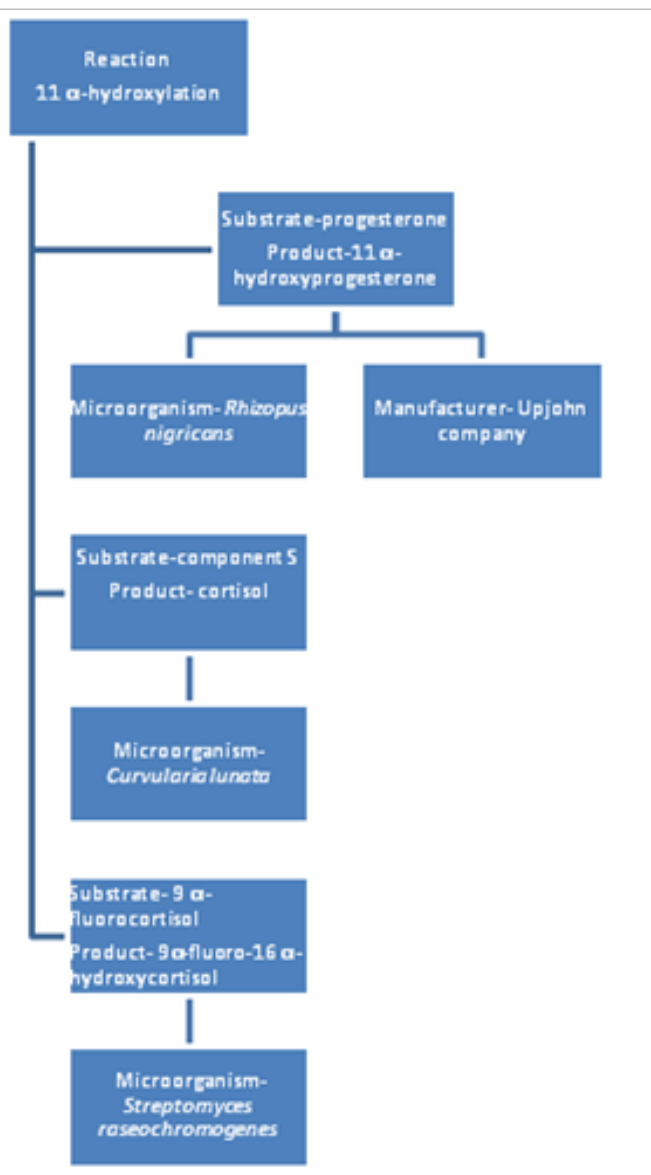

Figure 2 Biotransformational II alpha-hydroxylation of important steroids. 
The breakdown product 3-hydroxy-9,10-secoandrostatriene-9,17dione is produced from cholesterol via an opening of the B ring, with the production of two useful intermediate products, androstendione and androstadiendione with the help of an enzyme named as Arthrobacter simplex.

\section{Transformation of non-steroid compounds \\ Dihydroxyacetone from glycerol}

Dihydroxyacetone has lots of significance. It is used in lotions and cosmetics. Various acetic acid bacteria such as Gluconobacter melanogenus used in this microbial transformation. ${ }^{21}$

\section{Prostaglandins}

These are unsaturated fatty C-20 fatty acids hormones. These contains various medical significance such as PGE-2 used as contraceptive, PEG-2 used for the alleviation of pain of child-birth, PEG-1 for the treatment of congenital heart failure and also for the treatment of digestive diseases. These prostaglandins can be produced from unsaturated fatty acids by microbial transformation with pathogenic fungi such as Cryptococcus neoformans. ${ }^{22}$

\section{L-Ascorbic acid (vitamin C)}

The process for the production of L-ascorbic acid is called Reichstein-Grussner synthesis. This process of microbial conversion consists of several steps and this L-ascorbic acid is used in vitamin preparation or as an antioxidant in food manufacture. The oxidation stage from D-sorbitol to L-sorbose is carried out by Acetobacter suboxydans in a submerged process at $30-35^{\circ} \mathrm{C}$ with vigorous stirring and aeration. This process is carried out continuously in two stages; the first step involves the oxidation of glucose by Erwinia species to 2,5-diketo-D-gluconic acid via D-gluconic acid and 2-keto-Dgluconic acid. The second step involves a reduction of 2,5-DKG to 2-keto-1-gulonic acid which is catalysed by a Corynebacterium species.

\section{Transformation of antibiotics}

The microbial transformation of existing antibiotics has been done with the objective of developing new, modified and improved antibiotics which contain many qualities like reduced toxicity, broad antimicrobial spectrum, enhanced oral adsorption, less resistant/ allergic effects. ${ }^{23}$ In most cases, any transformation step causes a partial or complete inactivation of antibiotic. Several typical examples of the many possible reactions are given here:-

i. Indirect transformation:- In controlled biosynthesis, altered antibiotics are produced in the presence of inhibitors or modified precursors in the medium. For example, Streptomyces parvulus produces two new actinomycins in which proline is replaced by cis-4-methylproline. ${ }^{24}$ New compounds have been found when mutants blocked in the synthesis of a particular antibiotic were used. Mutational synthesis can lead to the generation of only few improved antibiotics such as 5-epi-sisomicin which has proved its sufficient efficacy to undergo clinical trials.

ii. Direct transformation: Hydrolysis of the functional groups led to inactivation of the antibiotics. But in the case of lankacidin-C-14-butyrate, a bioconversion product formed from lankacidin $\mathrm{C}$ and methyl-butyrate by Bacillus megaterium IFO 12108, improved antimicrobial activity with lower toxicity was obtained.

\section{Transformation of pesticides}

Agents for plant disease and pest control are necessary for the survival of the world's population. High stability of the compounds used is vital for the vector control programs, but this stability has a negative effect on the environment..$^{25}$

In this aspect, microbial transformation is of interest not for the production of new active agents, but for the greatest possible detoxification of the environment. This involves enzymatic conversions of xenobiotics. Removal of xenobiotics from ecosystem can be accomplished through various mechanisms.

\section{Metabolism}

Xenobiotics can serve as substrates for microbial growth and energy production. ${ }^{26}$ Complete breakdown of some substrates to carbondioxide and water. For example:- herbicide dalapon (a chlorinated fatty acid) is converted by Arthrobacter species into pyruvate (Figure 3 ).

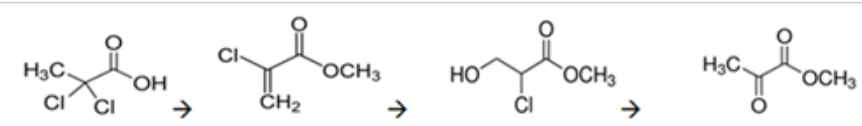

$$
\begin{aligned}
& \text { Dalapon 2-chloroacrylate 2-chloro-2-hydroxy-propionate pyruvate }
\end{aligned}
$$

Figure 3 Microbial transformation of Dalapon to pyruvic acid.

\section{Cometabolism}

Cometabolism normally causes mere modification of molecules, which may result in either a decrease or an increase in toxicity. Microorganisms involved in it do not obtain energy from the transformation reaction and require another substrate for the growth. Complete breakdown of a compound can be achieved through the combined action of different organisms. For example:- Dehalogenation reactions are important cometabolism reactions which may make pesticide molecules accessible for further breakdown (Figure 4). Some compounds such as chlordecone, a hexachlorocyclopentadiene derivative with excellent insecticide effects, are not easily attacked by microorganisms because of their complicated structure and high degree of halogenation.

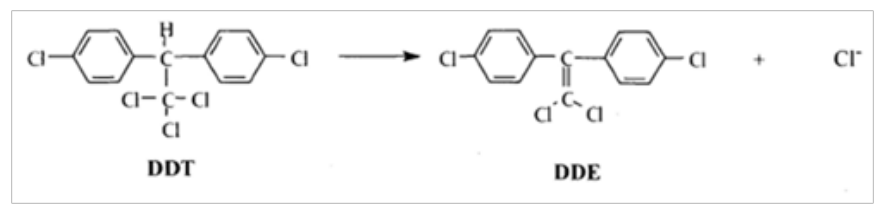

Figure 4 Conversion of DDT to DDE by Dehydrochlorination.

\section{Transformation of pollutants}

In recent years, interests in the biotransformation of various pollutants using microorganisms have gathered much momentum in order to clean up the polluted environment. ${ }^{27}$ The catabolic diversity of microbes are been utilized for the bioremediation of a huge range of compounds including polyaromatic hydrocarbons (PAHs), pharmaceutical substances, radionuclides, hydrocarbons (e.g. oil) and polychlorinated biphenyls (PCBs). The main disturbing trend is the toxicity threat these pollutants pose to the public health. Major advance in the molecular techniques, bioinformatics, genomic and metagenomics are being used to amplify the transformation of xenobiotic compounds. ${ }^{28}$ Although most organisms have detoxifying 
abilities, microbes especially bacteria has an important part to play in this process. High growth rate, metabolic diversity and horizontal gene transfer help them to grow and adjust according to the ever changing environmental conditions.

Both aerobic and anaerobic bacterial genera found have been associated with the biotransformation of a wide range of xenobiotic chemicals. Bacillus, Pseudomonas, Escherichia, Rhodococcus, Gordonia, Moraxella, Micrococcus are members of the aerobic genera while the anaerobic types includes Methanospirillum, Pelatomaculum, Syntrophobacter, Desulfotomaculum, Syntrophus, Desulfovibrio and Methanosaeta..$^{29}$ Mycobacterium vaccae have been demonstrating the capabilities to catabolize acetone, cyclohexane, styrene, benzene, ethylbenzene, propylbenzene, dioxane, and 1,2-dichloroethylene. Pseudomonas and Bacillus are known to degrade PCB (Polychlorinated Biphenyls) very efficiently. Some strains of Pseudomonas, Acetobacter and Klebsiella have also been able to bio-fix carcinogenic azo compounds. It is found that Pseudomonas $\mathrm{BCb} 12 / 1$ and $\mathrm{BCb} 12 / 3$ have exceptional degradation capabilities of low ethoxylated NPnEO (Non phenol Polyethoxylates). ${ }^{30}$

Anaerobicmethanogens(Methanospirillumhungatei,Methanosaeta concilii, Syntrophobacter fumaroxidens) are mainly involved in the degradation of Phthalate compound. ${ }^{31,32}$ Recently Cunninghamella elegans, Pseudomonas knackmussii and P. pseudoalcaligenes KF707 have shown the ability to biotransform potential pollutants bearing the pentafluorosulfanyl $\left(\mathrm{SF}_{5}-\right.$ ) functional group. ${ }^{33}$

\section{Petroleum biotransformation}

Petroleum hydrocarbons are the driving force of our industry and daily life. ${ }^{34}$ However, one of the major concerns these days is the hydrocarbon contamination resulting from the activities related to the petrochemical industry. ${ }^{35}$ Leaks and accidental release are the main source of soil and water pollution. Petroleum oil is carcinogenic and neurotoxic for all living forms.$^{36}$ So various mechanical and chemical methods have been employed for soil and water remediation. ${ }^{37}$ But, bioremediation using microbes is the most efficient method to detoxify the pollutants because it leads to complete mineralization and is cost effective.

Many aquatic and marine microflora have been shown to play an important role in the biodegradation of oil spills by breaking down oil contaminants into nontoxic forms. ${ }^{38}$ Biotransformation of petroleum hydrocarbons is carried out mainly by bacteria, yeast and fungi. ${ }^{39}$ Jones et al., ${ }^{40}$ have reported the presence of biodegraded petroleum -derived aromatic hydrocarbons in marine sediments. ${ }^{40}$ Daugulis and McCracken have reported the degradation of polyaromatic hydrocarbons by Sphingomonas. ${ }^{41}$

Bacterial genera such as Rhodococcus, Pseudomonas, Arthrobacter, and Mycobacterium are active degraders of alkylaromatic degradation of petroleum hydrocarbons. Bacterial genera, namely, Gordonia, Brevibacterium, Corynebacterium sp., Flavobacterium sp., Pseudomonas fluorescens, P. aeruginosa, Actinocorallia, Klebsiella, Rhizobium, Bacillus sp and Alcaligenes sp, Aeromicrobium, Dietzia, Burkholderia and Mycobacterium have been isolated from petroleum contaminated sites and they demonstrated to be the potential role players for hydrocarbon degradation. ${ }^{42}$

Fungal genera namely Aspergillus, Penicillium, Talaromyces, Amorphoteca, Neosartorya, Cephalosporium have been isolated from petroleum sites and were noted to play an important role in bioremediation of oil spills. ${ }^{43}$

\section{Conclusion}

Biotransformation is the basis of life. Microbes have been widely applied for steroid biotransformation to prepare specific derivatives, the production of which is difficult by traditional synthetic methods. Biotransformation is also good to handle the environmental problems like degradation of xenobiotics and petroleum hydrocarbons as they are real world problem. Therefore, based on the present review, it may be concluded that microbial biotransformation is a boon for the current world with its wide range of applications.

\section{Acknowledgements}

None.

\section{Conflict of interest}

The author declares no conflict of interest.

\section{References}

1. Asha S, Vidyavathi M. Cunninghamella - a microbial model for drug metabolism studies - a review. Biotechnol Adv. 2009;27(1):16-29.

2. Pervaiz I, Ahmad S, Madni MA, et al. Microbial biotransformation: a tool for drug designing (Review). Prikl Biokhim Mikrobiol. 2013;49(5):435449.

3. Cresnar B, Petric S. Cytochrome P450 enzymes in the fungal kingdom. Biochim Biophys Acta. 2011;1814(1):29-35.

4. Bianchini LF, Arruda MFC, Vieira SR, et al. Microbial biotransformation to obtain new antifungals. Front Microbiol. 2015;6:1433.

5. Biotransformation of xenobiotics.

6. Jones AL, Fawcett DW. Hypertrophy of the granular endoplasmic reticulum in hamster liver induced by phenobarbital (with a review on the functions of this organelle in liver). J Histochem Cytochem. 1966;14(3):215-232.

7. Karigar CS, Rao SS. Role of microbial enzymes in the bioremediation of pollutants: a Review. Enzyme Res. 2011;2011:11.

8. Parkinson A. Biotransformation of xenobiotics. In: Klaassen CD, editor. Toxicology: the basic science of poisons. Access Pharmacy, 2001. p. 133-224.

9. Kebamo S, Tesema S, Geleta B. The role of biotransformation in drug discovery and development. J Drug Metab Toxicol. 2015;6:196.

10. Huttel W, Hoffmeister D. Fungal biotransformations in pharmaceutical sciences. In: Hofrichter M, editor. Industrial applications, the mycota $X$, 2. Germany: Springer; 2010. p. 293-317.

11. Coelho MA, Ribeiro BD. White Biotechnology for Sustainable Chemistry. UK: RCS publishing; 2015.

12. Hegazy ME, Mohamed TA, ElShamyAI, et al. Microbial biotransformation as a tool for drug development based on natural products from mevalonic acid pathway: A review. $J$ Adv Res. 2015;6(1):17-33.

13. Chibata I, Wingard LB. Applied Biochemistry and Bioengineering. Enzyme Technology. 1983;1:1-355.

14. Foody P. Method for increasing the accessibility of cellulose in lignocellulosic materials, particularly hardwoods agricultural residues and the like. USA; 1984.

15. Stankiewicz AI, Jacob AM. Process intensification: transforming chemical engineering. Chem Eng Prog. 2000;96:22-34.

16. Sultan A, Rauf Raza A. Steroids: a diverse class of secondary metabolites. Med chem. 2015;5:310-317. 
17. Okafor N. Modern industrial microbiology and biotechnology. New Hamsphire: Science Publishers; 2007.

18. Kime DE. The Steroids. In: Chester-Jones I, Ingleton PM, editors Fundamentals of comparative vertebrate endocrinology. Germany: Springer; $1987.14 \mathrm{p}$.

19. Trost BM, Matthew LC. Asymmetric transition-metal-catalyzed allylic alkylations: applications in total synthesis. Chem Rev. 2003;103(8):29212944.

20. Samanta TB, Roy N, Chattopadhyay S. An improved 11 alphahydroxylation of progesterone by Aspergillus ochraceus TS. Biochem J. 1978;176(2):593-594

21. Gupta A, Singh VK, Qazi GN, et al. Gluconobacter oxydans: its biotechnological applications. $J$ Mol Microbiol Biotechnol. 2001;3(3):445-456.

22. Tsitsigiannis DI, Bok JW, Andes D, et al. Aspergillus cyclooxygenaselike enzymes are associated with prostaglandin production and virulence. Infect Immun. 2005;73(8):4548-4559.

23. Jobanputra AH, Patil GD, Sayyed RZ, et al. Microbial transformation of rifamycin: A novel approach to rifamycin derivatives. Ind J Biotechnol. 2003;2:370-377.

24. Katz E, Williams WK, Mason KT, et al. ovel actinomycins formed by biosynthetic incorporation of cis-and trans-4-methylproline. Antimicrob agents chemother. 1977;11(6):1056-1063.

25. Bollag JM. Microbial transformation of pesticides. Adv Appl Microbiol. 1974;18:75-130.

26. Boopathy R. Factors limiting bioremediation technologies. Bioresour Technol. 2000;74(1):63-67.

27. Pajouhesh H, George R. Medicinal chemical properties of successful central nervous system drugs. NeuroRx. 2005;2(4):541-553.

28. Sinha S, Chattopadhyay P, Pan L, et al. Microbial transformation of xenobiotics for environmental bioremediation. Afr J Biotechnol. 2009;8(22):6016-6027.

29. Chowdhury A, Pradhan S, Saha M, et al. Impact of pesticides on soil microbiological parameters and possible bioremediation strategies. $J$ Ind Microbiol. 2008;48(1):114-127.

30. DiGioia D, Michelles A, Pierini M, et al. Selection and characterization of aerobic bacteria capable of degrading commercial mixtures of lowethoxylated nonylphenols. J Appl Microbiol. 2008;104(1):231-242.
31. Qiu YL, Sekiguchi Y, Imachi $\mathrm{H}$, et al. Identification and isolation of anaerobic, syntropic phthalate isomer degrading microbes from methanogenic sludges treating wastewater from terepthalate manufacturing. Appl Environ Microbiol. 2004;70(3):1617-1626.

32. Zhang $\mathrm{C}$, Bennet GN. Biodegradation of xenobiotics by anaerobic bacteria. Appl Microbiol Biotechnol. 2005;67(5):600-618.

33. Kavanagh E, Winn M, Gabhann CN, et al. Microbial biotransformation of aryl sulfanylpentafluorides. Environ Sci Pollut Res Int. 2014;21(1):753758.

34. Mathew H. Refined politics: Petroleum products, neoliberalism, and the ecology of entrepreneurial life. Journal of American Studies. 2012;46(2):295-312.

35. Das N, Chandran P. Microbial degradation of petroleum hydrocarbon contaminants: an overview. Biotechnol Res Int. 2010;2011:941810.

36. O Peter Abioye. Biological remediation of hydrocarbon and heavy metals contaminated soil. In: Simone P, editor. Soil Contamination. 2011.

37. Masciangioli T, Zhang W. Peer reviewed: environmental technologies at the nanoscale. Environ Sci Technol. 2003;37(5):102A-108A.

38. McGenity TJ, Folwell BD, McKew BA, et al. Marine crude-oil biodegradation: a central role for interspecies interactions. Aquat Biosyst. 2012;8(1):10.

39. Atlas RM. Microbial degradation of petroleum hydrocarbons: an environmental perspective. Microbiol Rev. 1981;45(1):180-209.

40. Jones DM, Douglas AG, Parkes RJ, et al. The recognition of biodegraded petroleum-derived aromatic hydrocarbons in recent marine sediments. Marine Pollution Bulletin. 1983;14(3):103-108.

41. Daugulis AJ, McCracken CM. Microbial degradation of high and low molecular weight polyaromatic hydrocarbons in a two-phase partitioning bioreactor by two strains of Sphingomonas sp. Biotechnol Lett. 2003;25(17):1441-1444.

42. Chaillan F, Le Flèche A, Bury E, et al. Identification and biodegradation potential of tropical aerobic hydrocarbon degrading microorganisms. Res Microbiol. 2004;155(7):587-595

43. Koul S, Fulekar MH. Petrochemical Industrial Waste: Bioremediation Techniques An Overview. International Journal of Advancements in Research \& Technology. 2013;2(7):1-47. 